\title{
Imaging of adult leukodystrophies
}

\author{
Imagem nas leucodistrofias do adulto \\ Claudia Costa Leite ${ }^{1,2}$, Leandro Tavares Lucato ${ }^{3}$, Germana Titoneli Santos ${ }^{3}$, Fernando Kok ${ }^{4}$, Anderson \\ Rodrigues Brandão ${ }^{4}$, Mauricio Castillo ${ }^{2}$
}

\begin{abstract}
Leukodystrophies are genetically determined white matter disorders. Even though leukodystrophies essentially affect children in early infancy and childhood, these disorders may affect adults. In adults, leukodystrophies may present a distinct clinical and imaging presentation other than those found in childhood. Clinical awareness of late-onset leukodystrophies should be increased as new therapies emerge. MRI is a useful tool to evaluate white matter disorders and some characteristics findings can help the diagnosis of leukodystrophies. This review article briefly describes the imaging characteristics of the most common adult leukodystrophies.
\end{abstract}

Keywords: leukodystrophies, adult form, magnetic resonance imaging, imaging.

RESUMO

Leucodistrofias são doenças geneticamente determinadas. Apesar das leucodistrofias afetarem principalmente crianças lactentes e infantes, estas doenças podem acometer a faixa etária adulta. Nos adultos, as leucodistrofias podem ter uma apresentação clínica e de imagem distinta daquela da infância. Um aumento na suspeita clínica de leucodistrofias com início tardio deve ocorrer associado ao aparecimento de novas alternativas terapêuticas. Este artigo de revisão descreve sumariamente as características de imagem nas leucodistrofias no adulto.

Palavras-chave: leucodistrofias, formas do adulto, ressonância magnética, métodos de imagem.

Leukodystrophies are genetically determined disorders that primarily affect the white matter. In recent years, advances in molecular genetics have made the characterization and diagnosis of many leukodystrophies possible with the identification of defective genes. Furthermore, the widespread use of magnetic resonance imaging (MRI) allows the identification of white matter changes even at the beginning of the disease, in pre-symptomatic patients and carriers.

Even though leukodystrophies essentially affect children and present in early infancy and childhood, these disorders may affect adults and many times, with a distinct clinical and imaging presentations other than those found in childhood. As leukodystrophies are a heterogeneous group of diseases, many pathological findings are common to all, such as demyelination, hypomyelination and vacuolization. Commonly the white matter involvement is bilateral and symmetric.

Adult leukodystrophies or late-onset leukodystrophies can manifest initially as isolated psychiatric symptoms such as cognitive decline, behavioral problems, memory loss, psychosis and dementia. Other clinical manifestations include motor signs, peripheral neuropathy, bulbar dysfunction and epilepsy. Furthermore, in adults, disease progression is slower in comparison to the childhood subtypes of the same leukodystrophy.

Clinical awareness of late-onset leukodystrophies should be increased, as new therapeutic approaches have been developed, such as stem cell transplantation, enzyme replacement and gene therapy ${ }^{1}$. MRI is a useful tool to evaluate new forms of leukodystrophies and to allow longitudinal studies of disease progression ${ }^{2,3,4}$. The aim of this review is to briefly describe the imaging characteristics of the most common adult leukodystrophies.

\section{IMAGING FINDINGS}

\section{X-linked adrenoleukodystrophy (X-ALD)}

$\mathrm{X}$-linked adrenoleukodystrophy (X-ALD) is associated with the accumulation of very long chain fatty acids (VLCFA) in serum, neural tissues and adrenal glands. The

${ }^{1}$ Departamento de Radiologia, Faculdade de Medicina, Universidade de São Paulo, Sao Paulo SP, Brazil;

${ }^{2}$ Radiology Department, School of Medicine, University of North Carolina, Chapel Hill North Carolina, United States;

${ }^{3}$ Instituto de Radiologia, Hospital das Clínicas, Faculdade de Medicina, Universidade de São Paulo, Sao Paulo SP, Brazil;

${ }^{4}$ Departamento de Neurologia, Faculdade de Medicina, Universidade de São Paulo, Sao Paulo SP, Brazil.

Correspondence: Claudia Costa Leite; Radiologia, Faculdade de Medicina USP; Av. Dr. Ovidio Pires Campos, s/n Inrad Portaria 5, Ressonância Magnética; 05403-010 São Paulo SP, Brasil.

Conflict of interest: There is no conflict of interest to declare.

Received 23 April 2014; Received in final form 07 May 2014; Accepted 27 May 2014. 

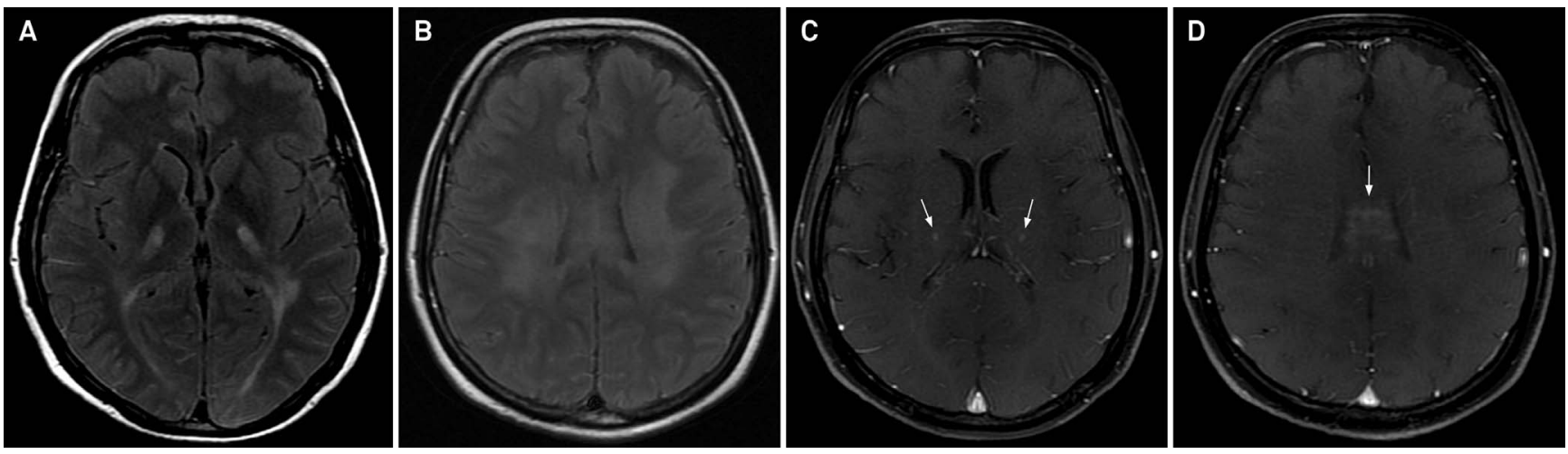

Figure 1. Adrenomyeloneuropathy with brain involvement in a 36-year-old male. Axial FLAIR (A and B) demonstrates bilateral hyperintensities in the deep white matter, body of the corpus callosum and internal capsules. Axial enhanced T1-weighted images (C and D) shows enhancement of the internal capsules and the body of the corpus callosum (arrows).

mutation associated to ALD-X is in the ABCD1 gene. The cell accumulation of VLCFA causes demyelination in the central nervous system (CNS) and cell destruction in the adrenal glands. As an X-linked inherited disease, $\mathrm{X}$-ALD affects mostly males. In adult males the predominant form is adrenomyeloneuropathy. ALD mutation may occur in women and they sometimes manifest milder symptoms ${ }^{5}$.

Adrenomyeloneuropathy is a slowy progressive disease that presents with adrenal insufficiency, distal axonopathy (predominantly affecting the corticospinal tracts and dorsal columns of spinal cord) and peripheral neuropathy ${ }^{1,3}$. The clinical presentation is usually spastic paraparesis. Brain MRI shows signs of involvement of the corticospinal tracts, dorsal columns, corpus callosum and periventricular white matter (Figure 1). Around 20\% of adrenomyeloneuropathy patients develop a more severe phenotype called adult cerebral adrenoleukodystrophy. Psychiatric signs, followed by motor signs, optic atrophy, epilepsy and death characterize this form of cerebral ALD.
As in childhood ALD, contrast enhancement can be seen in the periphery of the white matter lesions ${ }^{3}$ (Figure 2). Cerebral adult form can manifest as asymmetric focal lesions sometimes mimicking a tumor, and in some reports has been related to previous traumatic episodes (Figure 3) 5 .

Heterozygous women are usually asymptomatic or present brisk tendon reflexes and impaired vibration sensation. An ill-defined pain syndrome may occur. In $15 \%$ of females progressive spastic paraparesis can ensue around 40 years of age. Very rarely, the X-ALD women present adrenal insufficiency or brain involvement. Brain MRI can be normal or be similar to that seen in adrenomyeloneuropathy $y^{3,5,7}$.

\section{Metachromatic leukodystrophy}

Metachromatic leukodystrophy is an autosomal recessive lysosomal disease caused by a deficiency of the enzyme arylsulfatase A (ARSA) that leads to the accumulation of sulfatides in oligodendrocytes, Schwann cells and some neurons causing
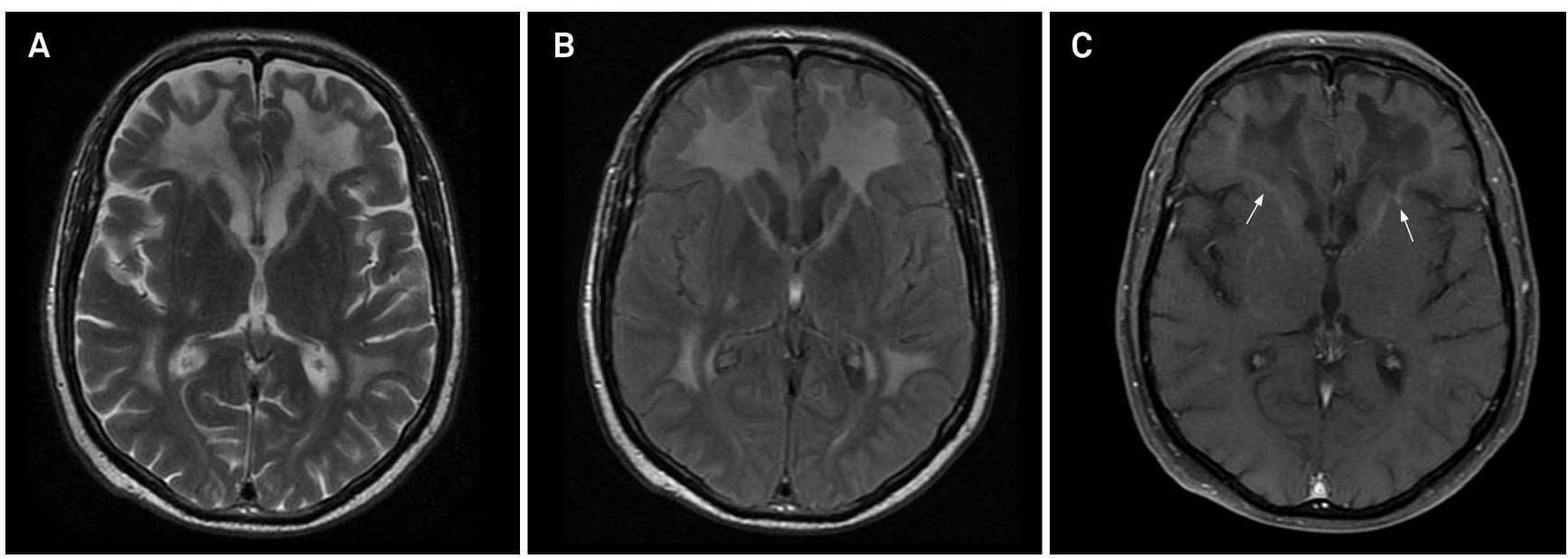

Figure 2. Adult cerebral leukodystrophy in a 54-year-old male with fast cognitive decline and pyramidal symptoms. Axial FLAIR and T2 (A and B) demonstrates bilateral periventricular, subcortical frontal and parieto-occipital white matter involvement. Note also hyperintensity in the internal capsules. Contrast enhanced axial T1-weighted images (C) shows faint enhancement in the periphery of the frontal lesions (arrows). 

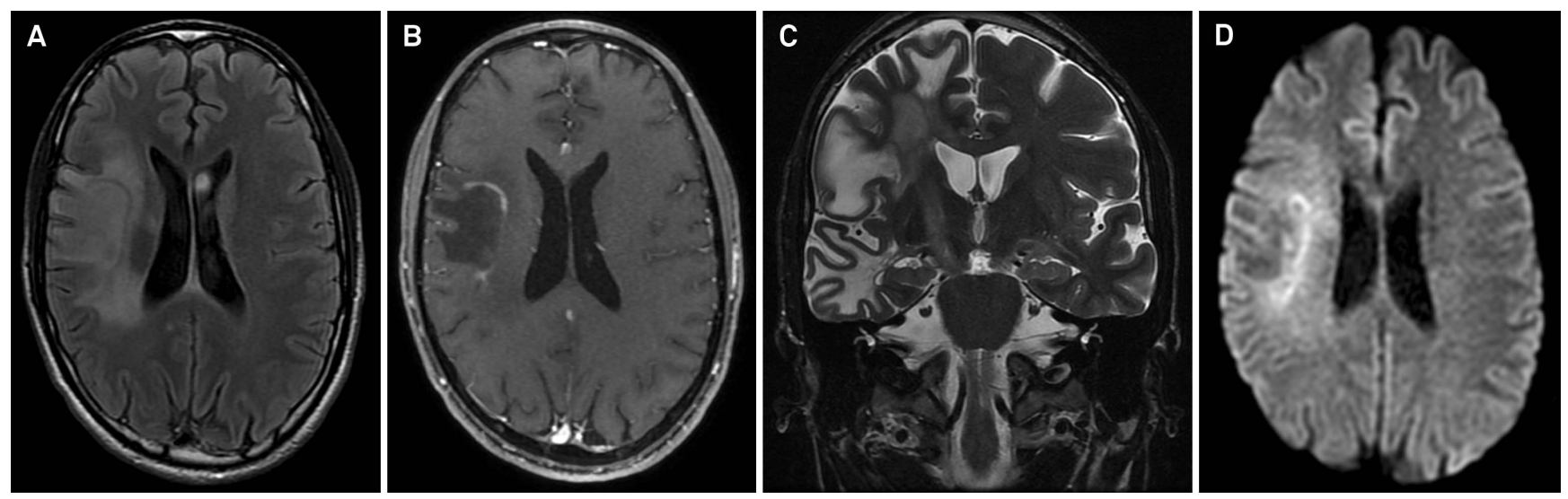

Figure 3. Adult cerebral leukodystrophy with an asymmetric presentation in a 28-year-old male who had a direct trauma to the right parietal region a few months earlier. Axial FLAIR (A) demonstrates a subcortical lesion in the right parietal lobe. Enhanced axial T1-weighted image (B) shows a peripheral discontinuous rim of enhancement. Coronal T2-weighted image (C) shows extension of the signal abnormality to the internal capsule. Axial diffusion-weighted image (D) shows a hyperintense rim in the lesion.

demyelination both in the central as well as in the peripheral nervous systems ${ }^{3}$. More than 100 mutations causing metachromatic leukodystrophy have been identified.

The adult-onset form, which occurs after 16 years of age, accounts for $20 \%$ of cases. The disease can manifest as late as the seventh decade of life ${ }^{2}$. In adulthood the main clinical presentations are psychiatric problems (cognitive, behavioral problems and memory deficits) sometimes mimicking schizophrenia followed by motor signs, such as spastic paraparesis, cerebellar ataxia and mild polyneuropathy. The disease course is self-limiting ${ }^{3,8}$.

MRI shows a bilateral periventricular leukoencephalopathy predominantly in the frontal regions, sparing the U-fibers in the initial phase of the disease. The corpus callosum can be affected. Cortical atrophy is also seen predominantly late in the course of the disease $e^{9,10,11}$.

\section{Krabbe's disease (Globoid cell leukodystrophy)}

Krabbe's disease is an autosomal recessive lysossomal storage disease caused by the deficiency of b-galactocerebrosidase (GALC) enzyme activity. The GALC defect leads to the accumulation of galactocerebroside and psychosine, which causes oligodendrocyte apoptosis and gliosis. The gene encoding for GALC is localized to chromosome 14q31. Unfortunately, neither the galactocerebrosidase activity nor the gene mutation can define the genotype in Krabbe disease ${ }^{12}$.

Adult Krabbe patients are older than 16 years, and the disease can manifest as late as in the fifth decade of life. Clinically late onset forms present pyramidal tract involvement with spastic paraparesis or tetraparesis. A peripheral demyelinating polyneuropathy occurs in up to $60 \%$ of patients, sometimes being asymmetric and involving bulbar muscles. Progressive cognitive decline, seizures and cortical blindness can also ensue. The disease progression is slow ${ }^{1,3,13}$.

On MRI, the deep supratentorial and cerebellar white matter are affected. Pyramidal tracts, splenium of corpus callosum and optic radiation can present hyperintensity on T2-weighted and FLAIR images. The signal abnormalities in the corticospinal tracts start in the perirolandic regions and extend to corona radiata, internal capsules, cerebral peduncles, ventral part of the pons and medulla bilaterally (Figure 4). Corpus callosum atrophy can also be seen. In rare occasions, MRI can be normal ${ }^{3,13}$.

\section{Alexander disease}

Alexander disease is a leukodystrophy characterized pathologically by diffuse Rosenthal fibers accumulation in astrocyte cytoplasm. The defect associated with this disease is located in the gene encoding for GFAP (glial fibrillary acid protein). After the identification of the gene defect, the number of adult-onset cases described in the literature has increased.

In Alexander disease, Rosenthal fibers are scattered throughout the cortex and in white matter and are most numerous in the subpial, perivascular and subependymal regions. The adult-onset form is diagnosed after 12 years of age up to the eighth decade of life. Clinical presentation includes a slowly progressive bulbar dysfunction (dysphagia, dysarthria, dysphonia), pyramidal signs and ataxia, with normal psychic and intellectual functions. When present, palatal myoclonus is suggestive of this diagnosis ${ }^{14,15,16}$.

MRI shows mild- to- severe atrophy of the medulla oblongata extending caudally to the cervical spinal cord, sometimes associated with signal abnormalities (Figure 5). Midbrain tegmentum atrophy has also been described, with preservation of the pontine base and this finding is quite specific of adult-onset Alexander disease ${ }^{17}$. Basal ganglia abnormalities (diffuse or patchy) have also been described, especially in patients under 40 years of age. In a few cases, periventricular garland that is typical of the juvenile form has been documented. Contrast enhancement is also reported in some patients especially in those under 40 years of age ${ }^{14}$. Relatives 

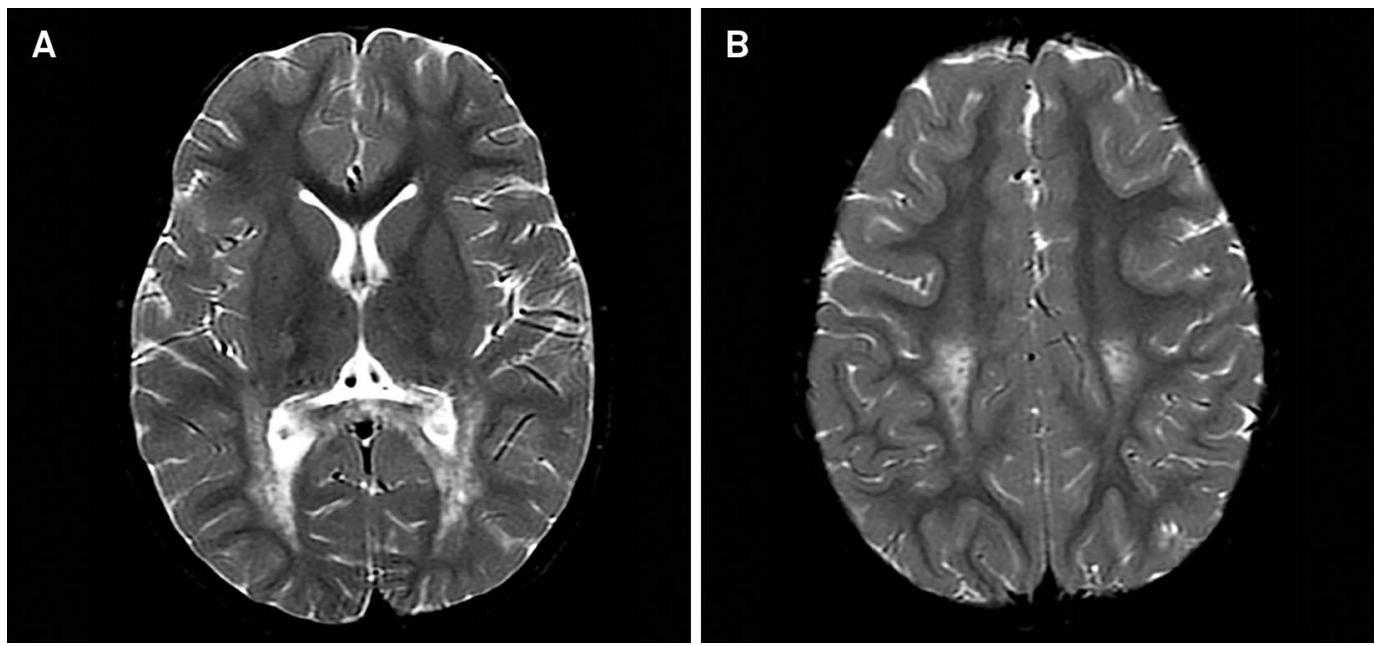

Figure 4. Late-onset Krabbe disease. Axial T2-weighted images (A and B) disclose bilateral and symmetric hyperintensity involving the corticospinal tracts from the centrum semiovale to the posterior limb of the internal capsule. There are similar changes appreciated in the splenium of corpus callosum.

with the gene mutation but without clinical manifestations (asymptomatic Alexander disease) can present a periventricular band of signal abnormality on MRI ${ }^{15}$.

\section{Vanishing white matter disease}

Vanishing white matter disease patients have mutations in the eIF2B (eukaryotic initiation factor 2B) complex genes. A mild variant of this disease has been described in adolescence and adulthood. The clinical onset in the juvenile or adult forms is characterized by migraine, psychiatric symptoms and dementia. Pseudobulbar palsy and progressive spastic paraparesis have also been described.

Women present ovarian insufficiency (dysgenesis) and leukodystrophy, a condition known as ovarioleukodystrophy. The ovarian failure may precede neurologic symptoms ${ }^{7,18}$.

MRI can show enlargement of the lateral ventricles and the white matter can be normal or present hyperintensity on T2-weighted and FLAIR images (Figure 6). Limited subcortical white matter hypointensity on FLAIR associated to the cystic breakdown or cavitation can be seen $^{19}$.

\section{Leukoencephalopathy with axonal spheroids and pigmented glia}

This very rare entity encompasses leukoencephalopathy with spheroids (HDLS) and pigmentary leukodystrophy (POLD), whether they represent the same disease or not is still controversial. They are hereditary diseases that unlike other leukodystrophies manifests exclusively in adults, most cases occurring in the 20- to 50-year age range. The clinical picture consists of behavioral changes, dementia, motor impairment (Parkinsonism, paraparesis or tetraparesis and ataxia) and epilepsy. There is an apparent autosomal dominant inheritance for HDLS and an autosomal recessive or dominant one for POLD ${ }^{2,20}$.

CT can show atrophy predominantly in the frontal lobes. MRI in HDLS shows frontal lobe atrophy and white matter lesions that can be diffuse, patchy or confluent and predominantly frontal or frontoparietal. The lesions are non-enhancing and hyperintense on T2-weighted images. Signal intensity abnormalities in the internal capsules and corticospinal tracts have also been described ${ }^{2,4,20}$.
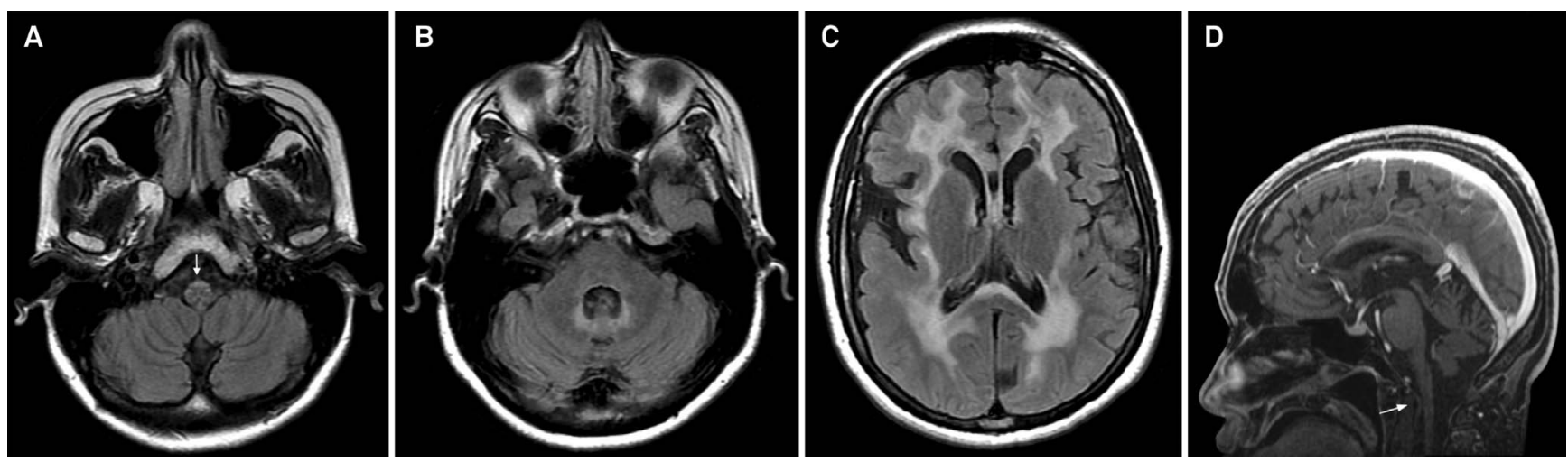

Figure 5. Adult-onset Alexander disease in a 47-year-old female. Axial FLAIR images shows atrophy of the medulla with hyperintensity in the projection of corticospinal tracts (arrow in A), bilateral hyperintensity in the deep cerebellar white matter (B) and in the cerebral hemispheres (C). Enhanced sagittal T1-weigted image (D) shows medulla and upper cervical cord atrophy (arrow). 

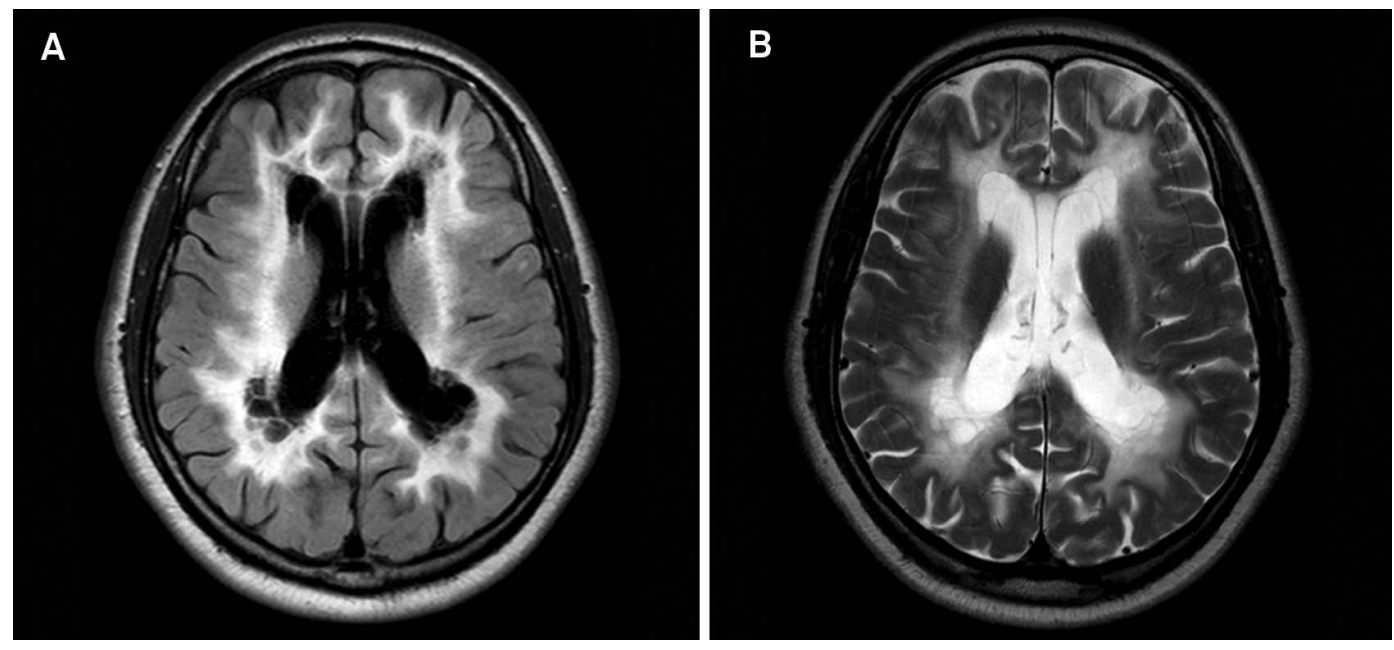

Figure 6. Vanishing white matter in a 48-year-old male. Axial T2-weighted (A) and FLAIR (B) images showing diffuse bilateral hyperintensity in the white matter extending to the $U$ fibers, internal and external capsules, with cavitations in the deep white matter

\section{Adult-onset autosomal dominant leukodystrophy (ADLD)}

Adult-onset autosomal dominant leukodystrophy (ADLD) is a very rare slowly progressive neurological disorder characterized by symmetric widespread myelin loss in the CNS. Patients usually present in the fourth to sixth decades of life with autonomic symptoms, bowel and bladder dysfunction, and orthostatic hypotension, later progressing to pyramidal symptoms and ataxia ${ }^{21}$. The gene that causes ADLD is located in chromosome 5q31. Two neuropathological observations make this leukodystrophy unique: preservation of oligodendroglia in the presence of subtotal demyelination and a lack of astrogliosis ${ }^{22}$.

The signal intensity abnormalities are most prominent in the frontoparietal white matter, cerebellar peduncles, corticospinal tracts and corpus callosum (Figure 7). The periventricular white matter is relatively spared. The changes in the uppermost corticospinal tracts underlying the motor cortex may represent the earliest radiologic imaging manifestation of the disease and also can be seen in asymptomatic family members. Extensive involvement of the cerebral white matter may be seen in the final stages of the disease. The MRI lesion pattern, in combination with the typical clinical symptoms and mode of inheritance, enable the diagnosis of $\mathrm{ADLD}^{23}$.

\section{Cerebrotendinous xanthomatosis}

Cerebrotendinous xanthomatosis is a rare autosomal recessive disease of the lipid metabolism. It is characterized by the deficiency of the mitochondrial enzyme sterol 27-hydroxylase (CYP27) that is involved in the synthesis of bile acids from cholesterol leading to the accumulation of cholesterol and cholestanol. Storage compounds can form xanthomata in various tissues. Initial symptoms occur in childhood with mental retardation, juvenile cataracts or chronic diarrhea. Progressive neurological deterioration occurs in adolescence or adulthood.

The clinical presentation includes psychiatric symptoms, spastic paraparesis, cerebellar ataxia, polyneuropathy. Tendon xanthomatas can be found, especially in the
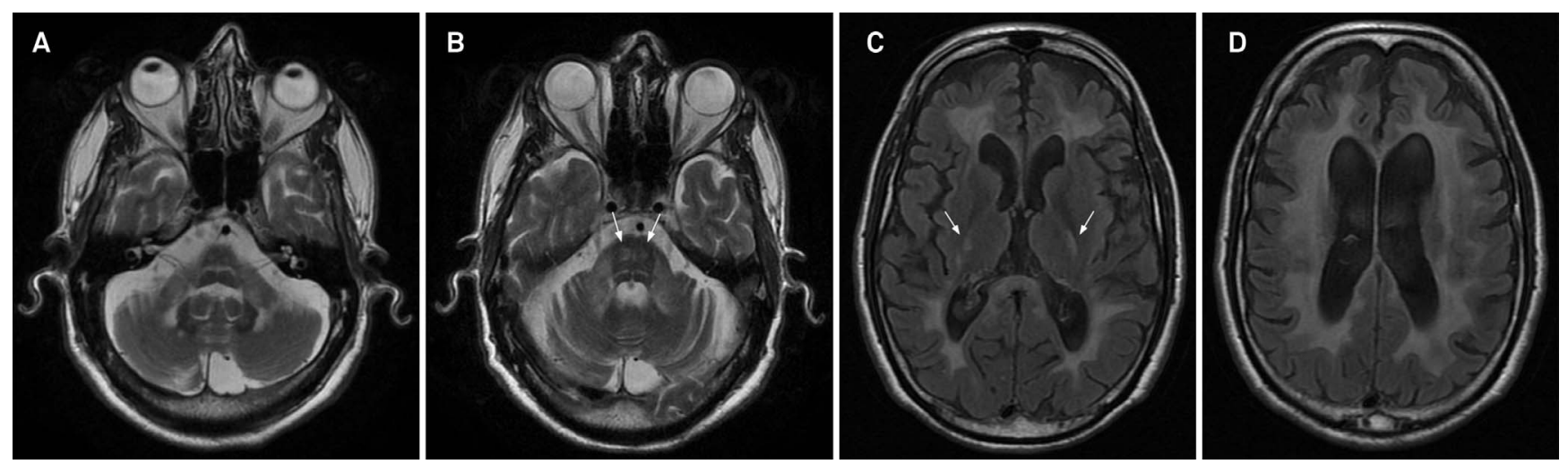

Figure 7. Adult-onset autosomal dominant leukodystrophy with autonomic symptoms (ADLD) in a 54-year-old man. Axial T2 images (A and B) shows high signal in the deep cerebellar white matter, middle cerebellar peduncles and pons. Axial FLAIR images ( $C$ and D) demonstrate diffuse hyperintensities in the periventricular and subcortical white matter, splenium of corpus callosum and atrophy. The corticospinal tracts show hyperintensity in the internal capsules (arrows in C) and pons (arrows in B). 

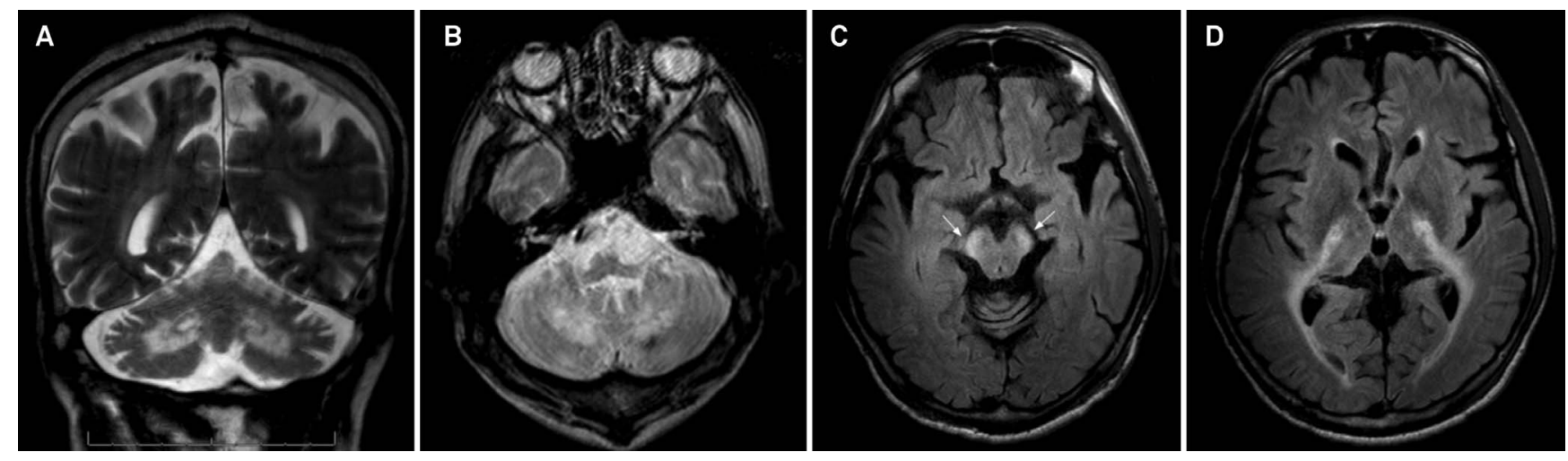

Figure 8. Cerebrotendinous xanthomatosis in a 47-year-old male with ataxia. Coronal T2-weighted imaging (A) demonstrates hyperintensity in the deep cerebellar white matter. Axial T2* (B) and FLAIR images ( $\mathrm{C}$ and D) show hyperintensity in the cerebellar hemispheres, cerebral peduncles (arrows in C), globus pallidus and deep cerebral white matter. Notice a hypointense area in B, seen in the left cerebellar hemisphere, a finding that can be appreciated eventually in the disease.

Achilles tendon ${ }^{3}$. Early treatment is important as patients can benefit from therapy ${ }^{24}$.

MRI shows hyperintensity on T2-weighted images in the dentate nucleus, cerebellar white matter (Figure 8), cerebellar peduncles, pyramidal tracts, periventricular white matter, corpus callosum and basal ganglia. In the late stages, hypointensity on T2-weighted images can be seen in the dentate nucleus ${ }^{3}$. Brain and cerebellar atrophy can also be $\operatorname{seen}^{24}$.

\section{Nasu-Hakola disease}

Nasu-Hakola disease (NHD) or polycystic lipomembranous osteodysplasia with sclerosing leukoencephalopathy is a rare, autosomal recessive disorder, caused by mutations in two genes: TREM 2 and DAP 12. NHD is characterized by a combination of diffuse bone cysts and pre-senile dementia. Most of the NHD patients first present in early adulthood with skeletal abnormalities (osseous phase). Neurological symptoms manifest in the fourth decade of life as psychiatric symptoms and memory loss (neuropsychiatric phase), which precedes the dementia phase. Progressive decline evolves to a profound dementia and death by the fifth decade of life ${ }^{25}$.

Bone imaging shows multiple cystic-like lesions leading to fractures in the wrists and ankles. In the brain, calcifications in the basal ganglia can be seen on CT, while cortical atrophy and nonspecific white matter involvement are best appreciated using MRI (Figure 9) ) $^{26,27}$.

\section{Cadasil and carasil}

CADASIL and CARASIL are small vessel diseases associated to ischemia and/or hemorrhage, diffuse white matter disease and vascular dementia ${ }^{28}$.

CADASIL is an acronym standing for cerebral autosomal dominant arteriopathy with subcortical infarcts and leukoencephalopathy; while CARASIL is similar, but is recessive instead of dominant.

CADASIL is caused by the mutation in NOTCH3 gene and affects adolescents and adults presenting with migraines, transient ischemic attacks, strokes, psychiatric
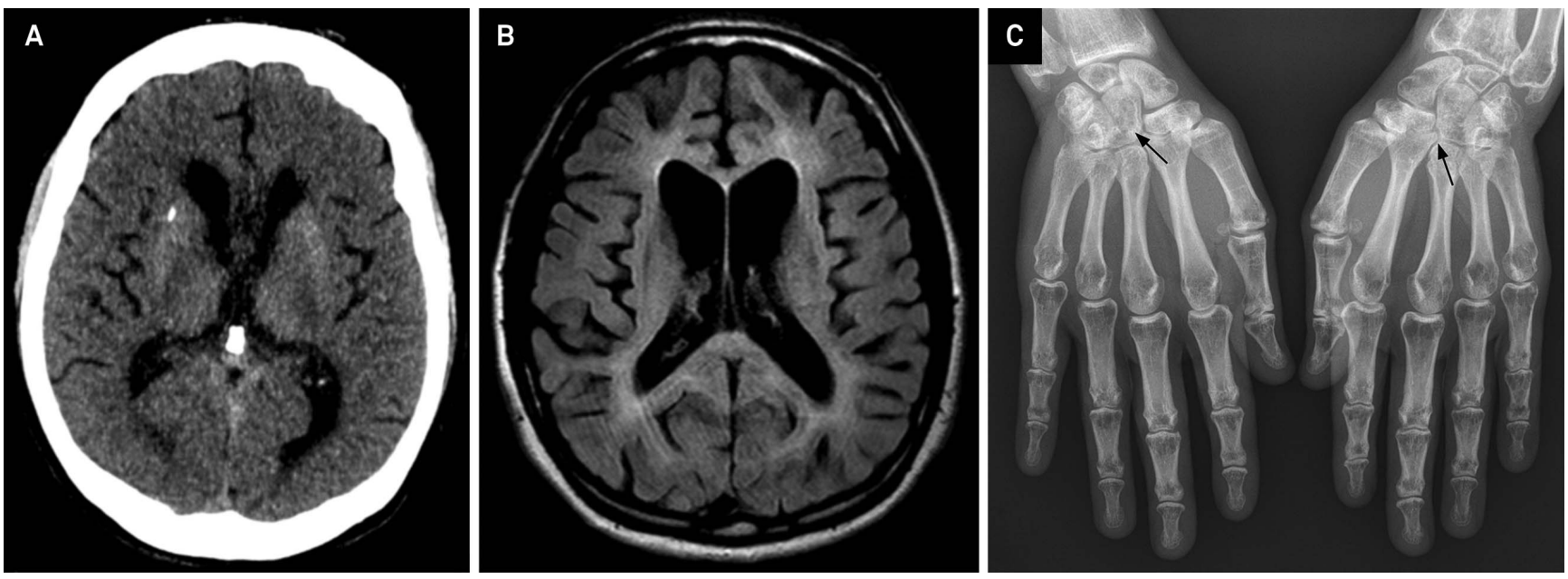

Figure 9. Nasu-Hakola disease in a 46-year-old man. Unenhanced CT (A) shows punctuate calcifications in the lentiform nuclei. Axial FLAIR image (B) demonstrates mild hyperintensity in the periventricular white matter. Note cortical atrophy. A radiograph of the hands (C) demonstrates cystic lesions in the carpal bones bilaterally (arrows). 

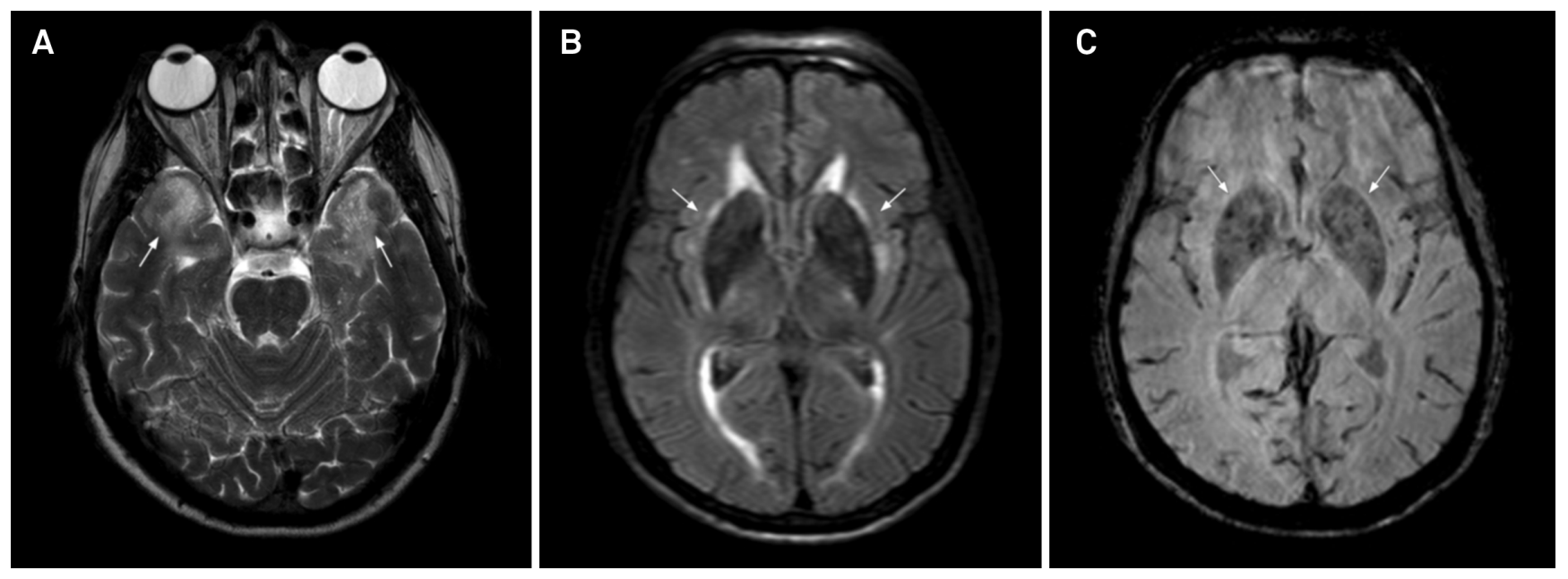

Figure 10. CADASIL in a 54-year-old male. Axial T2-weighted image (A) shows white matter hyperintense lesions in the temporal poles (arrows). Axial FLAIR image (B) shows hyperintensity in the periventricular white matter and external capsule (arrows). Note hypointensity in the lentiform nucleus. Axial SWI (susceptibility-weighted image - C) demonstrates some hypointense foci in the basal ganglia that may represent microbleeds (arrows).

disorders and cognitive decline. CADASIL is the most common cause of inherited vascular dementia in adults.

White matter involvement is usually found in the external capsules and anterior temporal poles, this last location being highly suggestive of CADASIL. The corpus callosum is less frequently involved. MRI changes precede the onset of symptoms by 10-15 years. On T2-weighted and FLAIR images there are round, predominantly periventricular lesions in the centrum semiovale, external capsules and anterior temporal poles (Figure 10). The signal changes tend to be symmetrical. Basal ganglia, thalamus, brainstem and corpus callosum can also be involved. Enlarged VirchowRobin spaces, small foci of restricted diffusion suggesting recent infarcts and microhemorrhage on $\mathrm{T}_{2} *$ images were described as well. The MRI abnormalities appear in the fourth decade of life and increase with age ${ }^{28,29}$.

CARASIL affects a younger population than CADASIL. The neurological symptoms are transient ischemic attacks and strokes. The MRI findings are diffuse white matter changes and lacunar infarcts ${ }^{30}$.

Other small vessel, genetically inherited disease, is the socalled autosomal dominant retinal vasculopathy with leukodystrophy and TREX1 mutation. It can present with white matter involvement in the brain and cerebellum and areas of contrast enhancement.
Other reported instances of adult-onset leukodystrophies include Pelizaeus-Merzbacher disease and vacuolating megalencephalic leukoencephalopathy with subcortical cysts. PelizaeusMerzbacher is rarely described in adults and the MRI findings include white matter diffuse T2-hyperintensities sparing small focal areas. MRI of patients with vacuolating leukoencephalopathy with subcortical cysts in adults shows bilateral extensive white matter changes with cysts in the temporal regions, sometimes the patients also present megalencephaly ${ }^{31,32}$.

Final remarks, leukodystrophies should be kept in mind in the differential diagnosis of bilateral white matter disease in adults. Some imaging findings can help suggest a final diagnosis. Involvement of the corticospinal tract can be seen in Krabbe's disease, adrenomyeloneuropathy, cerebrotendinous xanthomatosis and ADLD. Contrast enhancement can be seen in the adult cerebral form of ALD and Alexander disease. In adult-onset Alexander disease atrophy and signal abnormalities can be seen in the medulla oblongata and cervical cord. Limited subcortical hypointensity on FLAIR images is present sometimes in ovarioleukodystrophy. Dentate nucleus and cerebellar white matter abnormalities can occur in cerebrotendinous xanthomatosis. Brain calcifications, white matter abnormalities and bone cysts are present in Nasu-Hakola disease. In CADASIL involvement of the external capsules and anterior temporal poles are characteristic.

\section{References}

1. Köhler, W. Leukodystrophies with late disease onset: an update. Curr Opin Neurol 2010;23:234-241.

2. Costello DJ, Eichler AF, Eichler FS. Leukodystrophies. Classification, Diagnosis and treatment. The Neurologist 2009;15:319-328.

3. Sedel F, Tourbah A, Fontaine B, et al. Leukoencephalopaties associated with inborn errors of metabolism in adults. J Inherit Metab Dis 2008;31:295-307.
Lyon G, Fattal-Valevsky A, Kolodny EH. Leukodystrophies. Clinical and genetic aspects. Top Magn Reson Imaging 2006;17:219-242.

5. Moser HW, Mahmood A, Raymond GV. X-linked adrenoleukodystrophy. Nature Clin Pract Neurol 2007;3:140-151.

6. Raymond GV, Seidman R, Monteith TS, et al. head trauma can initiate the onset of adreno-leukodystrophy. J Neurol Sci 2010;290:70-74. 

Opin Neurol 2004;17:187-192.

8. Gleselmann V, Krägeloh-Mann I. Metachromatic leukodystrophy - an update. Neuropediatrics 2010;41:1-6.

9. Chebel S, Barboura I, Boughammoura-Bouatay A, et al. Adult-type metachromatic leukodystrophy mimicking multiple sclerosis. Can J Neurol Sci 2009;36:521-523.

10. Eichler F, Grodd W, Grant E, et al. Metachromatic leukodystrophy: a scoring system for brain MR imaging observations. AJNR 2009;30:1893-1897.

11. Martin A, Sevin C, Lazarus C, et al. Toward a better understanding of brain lesions during metachromatic leukodystrophy evolution. AJNR 2012;33:1731-1739.

12. Abdelhalim AN, Alberico RA, Barczykowski AL, et al. Patterns of magnetic resonance abnormalities in symptomatic patients with Krabbe disease correspond to phenotype. Pediatr Neurol 2014;50:127-134.

13. Debs R, Froissart R, Aubourg P, et al. Krabbe disease in adults: phenotypic and genotypic update from a series of 11 cases and review. J Inherit Metab Dis 2013;36:859-868.

14. Pareyson D, Fancellu R, Mariotti C, et al. Adult-onset Alexander disease: a series of 11 unrelated cases with review of the literature. Brain 2008;131:2321-2331.

15. Sawaishi Y. Review of Alexander disease: beyond the classical concept of leukodystrophy. Brain Dev 2009;31:493-498.

16. Balbi P, Salvini S, Fundarò C, et al. The clinical spectrum of lateonset Alexander disease: a systematic literature review. J Neurol 2010;257:1955-1962.

17. Namekawa $\mathrm{Y}$, Takiyama $\mathrm{Y}$, Honda J, et al. Adult-onset Alexander disease with typical "tadpole" brainstem atrophy and unusual bilateral basal ganglia involvement: a case report and review of the literature. BMC Neurology 2010;10:21-27.

18. Bugiani M, Boor I, Powers J, et al. Leukoencephalopathy with vanish white matter: a review. J Neuropathol Exp Neurol 2010;69:987-996.

19. Schiffmann R, Elroy-Stein O. Childhood ataxia with CNS hypomielination/vanish white matter disease- a common leukodystrophy caused by abnormal control of protein synthesis. Mol Genet Metabol 2006;88:71-75.
20. Wider C, Van Gerpen JA, Wszolek ZK. Leukoencephalopathy with spheroids (HDLS) and pigmentary leukodystrophy (POLD). A single entity? Neurology 2009;72:1953-1959.

21. Finnsson J, Melberg A, Raininko R. 1H-MR spectroscopy of adultonset autosssomal dominant leukodystrophy with autonomic symptoms. Neuroradiology 2013;55:933-939.

22. Coffeen CM, Mckenna CE, Koeppen AH, et al. Genetic localization of an autosomal dominant leukodystrophy mimicking chronic progressive multiple sclerosis to chromosome 5q31. Hum Mol Genet 2000;9:787-793.

23. Melberg A, Hallber L, Kalimo H, Raininko R. MR characteristics and neuropathology in adult-onset autosomal dominant leukodystrophy with autonomic symptoms. Am J Neuroradiol AJNR 2006;27:904-911.

24. Pudhiavan A, Agrawal A, Chaudhari S, Shukla A. Cerebrotendineous xanthomatosis- the spectrum of imaging findings. J Radiology Case Rep 2013;7:1-9.

25. Klünemann $\mathrm{HH}$, Ridha $\mathrm{BH}$, Magy L, et al. The genetic causes of basal ganglia calcification, dementia, and bone cysts: DAP12 and TREM2. Neurology 2005;64:1502-1507.

26. Bock V, Botturi A, Gaviani P, et al. Polycystic lipomembranous osteodysplasia with sclerosing leukoencephalopathy (PLOSL): a new report of an Italian woman and review of the literature. J Neurol Sci 2013;326:115-119.

27. Kilic SA, Oner AY, Yuce C, Ozlu IC. Imaging findings of Nasu-Hakola disease: a case report. Clin Imaging 2012;36:877-880.

28. Federico A, Di Donato I, Di Palma C, Taglia I, Dotti MT. Hereditary small vessel diseases: a review. J Neurol Sci 2010;322:25-30.

29. Chabriat $H$, Joutel A, Dichgans M, et al. CADASIL. The Lancet Neurology 2009;8:643-653.

30. Fukutake T. Cerebral autosoma recessive arteriopathy with subcortical infarcts and leukoencephalopathy (CARASIL): from discovery to gene identification. J Stroke Cerebrovascular Dis 2011;20:85-93.

31. Sasaki A, Miyanaga K, Ototsuji M, et al. Two autopsy cases with Pelizaeus- Merzbacher disease phenotype of adult onset, without mutation of proteolipid protein gene. Acta Neuropathol 2000;99:7-13.

32. Itoh N, Maeda M, Naito Y, et al. An adult case of megalencephalic leukoencephalopathy with subcortical cysts with S93L mutation in MLC1 gene: a case report and diffusion MRI. Eur Neurol 2006;56:243-245. 